\title{
Improvement of the rheological properties of trans-1,4-polyisoprene from Eucommia ulmoides Oliver by tri-branched poly(ricinoleic acid)
}

\author{
Bo-xing Zhang ${ }^{1}$, Jun-ichi Azuma ${ }^{1}$, Shinya Takeno ${ }^{2}$, Nobuaki Suzuki ${ }^{2}$, Yoshihisa Nakazawa ${ }^{2}$ and \\ Hiroshi Uyama ${ }^{1}$
}

This study investigated fully bio-based blends of trans-1,4-polyisoprene from Eucommia ulmoides Oliver (EuTPI) and linear poly (ricinoleic acid) (LPRA) or tri-branched poly(ricinoleic acid) (BPRA). LPRA and BPRA were synthesized using an immobilized lipase as the catalyst. Blends of EuTPI with LPRA or BPRA in various mixing ratios were examined. Scanning electron microscope observations suggest that BPRA was uniformly distributed in the EuTPI matrix in contrast to the poor distribution of LPRA, and BPRA was effectively incorporated into the crosslinked network of EuTPI by curing with dicumyl peroxide.

Rheological studies indicate that BPRA was more effective than LPRA at improving the processability of EuTPI. The storage modulus, loss modulus and complex viscosity $(\eta)$ of EuTPI over a frequency $(\omega)$ range from 0.1 to $100 \mathrm{rad} \mathrm{s}^{-1}$ at $80^{\circ} \mathrm{C}$ clearly decreased with an increase in BPRA content. At low frequency, the rheological behavior of EuTPI changed from that of an elastic gel to that of a liquid when 15 wt $\%$ of BPRA was added. Moreover, $\eta$ dramatically decreased to $\sim 5 \%$ of that of the pure EuTPI at $\omega=0.1 \mathrm{rad} \mathrm{s}^{-1}$. Thermogravimetric analysis results suggest that the blending of BPRA would not compromise the thermal stability of EuTPI. BPRA was found to promote the formation of more stable $\alpha$-phase crystallites in EuTPI by differential scanning calorimetry analysis.

Polymer Journal (2016) 48, 821-827; doi:10.1038/pj.2016.30; published online 16 March 2016

\section{INTRODUCTION}

Trans-1,4-polyisoprene is a semi-crystalline polymer that is harder and less elastic than its stereoisomer cis-1,4,-polyisoprene (CPI, the main component of natural rubber). ${ }^{1,2}$ Trans-1,4-polyisoprene can be extracted from a small number of plant species, such as Eucommia ulmoides, Palaquium gutta, Manilkara bidentata, Achras zapota, Garrya flavescens and G. wrightii. ${ }^{3-5}$ Among them, E. ulmoides is extensively cultivated in China as a source of a Chinese traditional medicine. ${ }^{5,6}$ Currently, there are several applications of natural trans-1,4-polyisoprene, for example, golf balls and endodontic-filling materials., ${ }^{2,7}$

In recent years, natural trans-1,4-polyisoprene extracted from E. ulmoides Oliver (EuTPI) has attracted researchers' interests because of an ever-increasing environmental awareness and fossil fuel crisis. Previously, we conducted a histochemical study of EuTPI accumulation in E. ulmoides Oliver, revealing that EuTPI was initially synthesized as granules in non-articulated laticifers and changed form to fibers along with laticifer maturation. ${ }^{3,6}$ We also developed a biobased polymer with a dynamically crosslinked network structure from EuTPI. ${ }^{8}$ Zhang and Xue ${ }^{9}$ studied the difference between EuTPI and synthetic trans-1,4-polyisoprene. Yan ${ }^{10}$ and Xue et al. ${ }^{11}$ developed a series of patented products based on EuTPI and also studied the damping properties of EuTPI and its blends with other elastomers.
Sarina et al. ${ }^{12}$ investigated the dynamic properties of EuTPI with different degrees of crosslinking.

One of the crucial problems of EuTPI is poor processability, which hampers its widespread application. Unlike CPI, EuTPI with high molecular weight $\left(M_{\mathrm{w}}>10^{6}\right)^{8}$ did not flow and still possessed a high modulus even above its melting point. This easily caused defects and failures during the extrusion and molding processes, which eventually affected the performance and appearance of the products. Thus, the improvement of the processability of EuTPI is an urgent issue.

Applications of ricinoleic acid have been extensively studied due to its abundance, excellent biodegradability and multi-functional groups. ${ }^{13-26}$ Ricinoleic acid, comprising $90 \%$ of castor oil, possesses hydroxyl and carboxyl groups, as well as a $\mathrm{C}=\mathrm{C}$ bond, making it polymerizable and crosslinkable. ${ }^{14}$ Ebata et al. ${ }^{19}$ synthesized a high molecular weight poly(ricinoleic acid) (PRA) from methyl ricinoleate (purity $>99 \%$ ) using an immobilized lipase as the catalyst. Such a polyester was a viscous liquid at room temperature, completely amorphous and biodegradable. Subsequently, they obtained a thermosetting elastomer from the vulcanization of PRA. ${ }^{18}$ Roberson et al. prepared a poly(L-lactide) (PLLA)/PRA diblock copolymer as a compatibilizer to improve the toughness of PLLA. ${ }^{16}$ Lebarbe et al. ${ }^{15}$ prepared a PLLA/PRA/PLLA triblock copolyester. By the incorporation

\footnotetext{
${ }^{1}$ Department of Applied Chemistry, Graduate School of Engineering, Osaka University, Suita, Japan and ${ }^{2}$ Hitz Research Alliance Laboratory, Graduate School of Engineering, Osaka University, Suita, Japan

Correspondence: Professor H Uyama, Department of Applied Chemistry, Graduate School of Engineering, Osaka University, 2-1 Yamadaoka, Suita, 565-0871, Japan.

E-mail: uyama@chem.eng.osaka-u.ac.jp

Received 12 September 2015; revised 8 January 2016; accepted 8 January 2016; published online 16 March 2016
} 

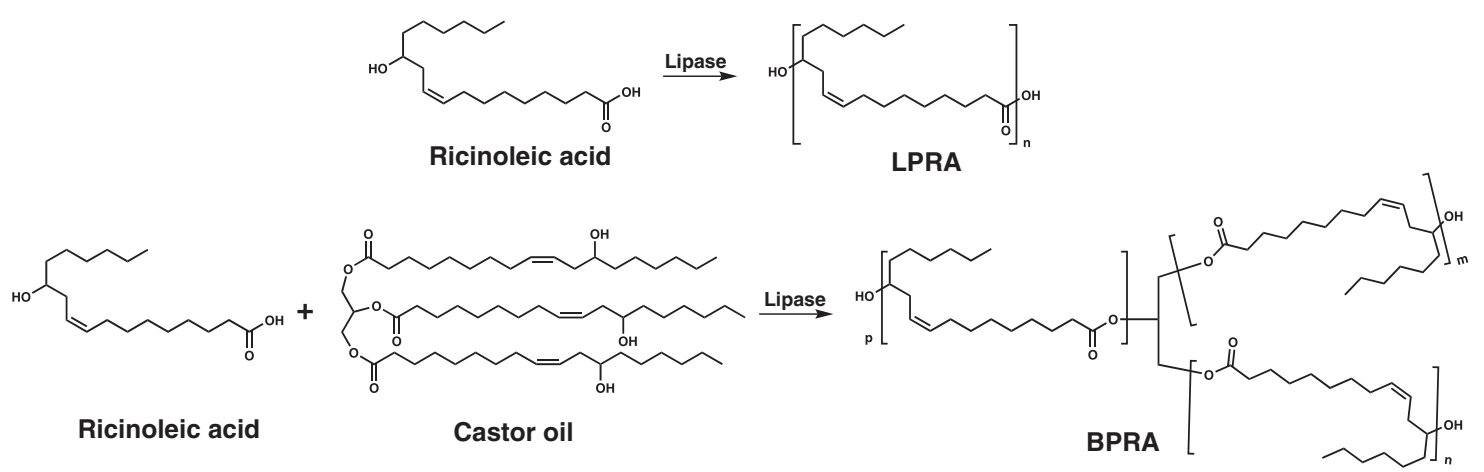

Figure 1 Scheme of the lipase-catalyzed synthesis of LPRA and BPRA. BPRA, tri-branched poly(ricinoleic acid); LPRA, linear poly(ricinoleic acid).

of $17 \mathrm{wt} \%$ of the PRA block, the ultimate strain increased from 5 to $98 \%$. Gautrot et al. ${ }^{17}$ synthesized a high molecular weight copolyester based on bile acid and ricinoleic acid via entropy-driven ring-opening metathesis polymerization. The copolyester displayed tunable mechanical properties and heterogeneous degradation behaviors. The aforementioned research incorporated the flexible and amorphous properties of PRA into rigid materials, such as PLLA and poly(bile acid). These previous studies inspired us to introduce PRA into EuTPI to improve the properties.

Herein, we synthesized two types of crosslinkable polyesters, a linear PRA (LPRA) and a tri-branched PRA (BPRA), as additives to improve the properties of EuTPI. LPRA was synthesized from cost-effective ricinoleic acid with an immobilized lipase as the catalyst. Similarly, BPRA was synthesized using castor oil as the molecular core (Figure 1). Various amounts of LPRA and BPRA were separately introduced into EuTPI. The morphology, crystallization behaviors, thermal and rheological properties of the blend films were systematically investigated. This research aims to improve the processability of EuTPI by blending it with a crosslinkable polyester.

\section{EXPERIMENTAL PROCEDURE}

\section{Materials}

Lipase (lipase PS IM Amano, immobilized on diatomaceous earth), ricinoleic acid (purity $\approx 80 \%$ ) and castor oil were purchased from Wako Pure Chemical Industries, Osaka, Japan. Dicumyl peroxide was purchased from Sigma-Aldrich, Ichikawa, Japan. Molecular sieves 4A 1/8 were purchased from Nacalai Tesque, Kyoto, Japan, and activated in vacuo at $150^{\circ} \mathrm{C}$ for $4 \mathrm{~h}$ before usage. All reagents, unless mentioned otherwise, were used as received.

EuTPI produced from the seeds of E. ulmoides Oliver was supplied by Hitachi Zosen, Osaka, Japan and purified according to a previous report. ${ }^{8}$ Briefly, $5 \mathrm{~g}$ of crude sample was dissolved in $300 \mathrm{ml}$ of toluene by vigorous stirring at $40^{\circ} \mathrm{C}$ for $48 \mathrm{~h}$. The undissolved impurity was removed by centrifugation. The pure EuTPI was obtained after evaporating the solvent under reduced pressure. The molecular weight of EuTPI $\left(M_{\mathrm{w}}=1.2 \times 10^{6}\right.$, polydispersity index $(\mathrm{PDI})=1.8$ ) was characterized by size exclusion chromatographic analysis.

\section{Synthesis of LPRA}

Ricinoleic acid $(2.0 \mathrm{~g}, 6.7 \mathrm{mmol})$ was added to a $100 \mathrm{ml}$ tube. Lipase PS (0.60 g, $30 \mathrm{wt} \%$ for ricinoleic acid) was pre-dried for $2 \mathrm{~h}$ in vacuo at room temperature and was carefully added at the bottom of the tube. ${ }^{19}$ The molecular sieves were sealed in the upper part of the tube (Supplementary Figure S1). The reaction was performed at $60^{\circ} \mathrm{C}$ for 7 days. Then, $20 \mathrm{ml}$ of chloroform was added to dissolve the product, which was followed by filtration to remove lipase PS. The solution was concentrated under reduced pressure, and the concentrate was dropped in $20 \mathrm{ml}$ of methanol to remove the unreacted monomer and oligomers. The product precipitated in the bottom layer and was collected and dried in vacuo at $60{ }^{\circ} \mathrm{C}$ until the weight was constant. The yield of LPRA was $\sim 80 \%$.

\section{Synthesis of BPRA}

Ricinoleic acid (2.0 g, $6.7 \mathrm{mmol})$ and castor oil $(0.16 \mathrm{~g}, 0.17 \mathrm{mmol})$ were added to a $100 \mathrm{ml}$ tube. The mixture was vigorously stirred at room temperature for $15 \mathrm{~min}$. Polymerization was carried out as described above for the synthesis of LPRA by using the pre-dried lipase PS as the catalyst $(0.65 \mathrm{~g}, 30 \mathrm{wt} \%$ for the total weight of ricinoleic acid and castor oil) and the molecular sieves as a water scavenger. After the reaction, the crude product was isolated by solubilization in chloroform $(20 \mathrm{ml})$, by the removal of lipase PS and by evaporation of the solvent under reduced pressure. The product was dissolved in $10 \mathrm{ml}$ of acetone by gentle heating. Then, the solution was sealed and kept overnight in an ice-water bath. The precipitate formed in the bottom layer and was collected. The purification procedures were performed twice. Finally, a transparent viscous liquid was obtained after drying to constant weight in vacuo at $60^{\circ} \mathrm{C}$. The yield of BPRA was $~ 70 \%$.

\section{Preparation of the films from EuTPI-LPRA and EuTPI-BPRA}

First, $0.050 \mathrm{~g}$ LPRA, $0.95 \mathrm{~g}$ EuTPI and $30 \mathrm{ml}$ of chloroform were sequentially added to a $50 \mathrm{ml}$ vial. Then, the mixture was vigorously stirred for $24 \mathrm{~h}$ at room temperature, which was followed by casting the resulting solution into a Teflon mold and drying it overnight. To completely remove the solvent, the sample was further dried in vacuo at room temperature for $24 \mathrm{~h}$. Then, the sample was sandwiched between two aluminium plates with a $100 \mu \mathrm{m}$ spacer. The hotpressing process was performed at $100^{\circ} \mathrm{C}$ under a pressure of $10 \mathrm{MPa}$ for $10 \mathrm{~min}$. Then, the film was naturally cooled down to room temperature. Finally, a blend film with $5 \mathrm{wt} \%$ of LPRA was prepared, which was referred to as EuTPI-LPRA5\%. According to the same procedure, EuTPI-LPRA10\%, EuTPI-LPRA15\%, EuTPI-LPRA20\%, EuTPI-BPRA5\%, EuTPI-BPRA10\%, EuTPI-BPRA15\% and EuTPI-BPRA20\% were prepared. For comparison, a film from the pure EuTPI was also prepared. The films were kept in vacuo for at least $24 \mathrm{~h}$ before any test.

The cured films were prepared using similar procedures except for the addition of dicumyl peroxide $(2.0 \mathrm{wt} \%$ for the total weight of BPRA and EuTPI) after EuTPI and BPRA were completely dissolved. ${ }^{9}$ The films were hotpressed at $150{ }^{\circ} \mathrm{C}$ under a pressure of $10 \mathrm{MPa}$ for $30 \mathrm{~min}$. The corresponding samples were referred to as EuTPI cured and EuTPI-BPRA15\% cured.

\section{Characterization}

${ }^{1} \mathrm{H}$ nuclear magnetic resonance (NMR) spectra were recorded in $\mathrm{CDCl}_{3}$ using a Bruker DPX-400 instrument (Bruker BioSpin, Billerica, MA, USA). Fourier transform infrared (FT-IR) spectra in the attenuated total reflection (ATR) mode were recorded on a Nicolet iS5 FT-IR spectrometer with an iD5 ATR accessory (Thermo Fisher Scientific, Waltham, MA, USA). Size exclusion chromatography was performed on a TOSOH SC-8020 apparatus equipped with refractive index and ultraviolet detectors (Tosoh, Tokyo, Japan). Two groups of columns, TSKgel $\mathrm{GMH}_{\mathrm{HR}}-\mathrm{H}$ (S) (for analysis of EuTPI) and TSKgel G4000H $\mathrm{HL}_{\mathrm{XL}}$ (for analysis of LPRA and BPRA), were used. Chloroform was used as the eluent at a flow rate of $1.0 \mathrm{ml} \mathrm{min}{ }^{-1}$ at $40{ }^{\circ} \mathrm{C}$. The molecular weight was calibrated by polystyrene standards. Thermogravimetric analysis was conducted on a SII TG/DTA7200 (Hitachi High-Tech Science, Tokyo, Japan) from 40 to $500{ }^{\circ} \mathrm{C}$ at a heating rate of $10^{\circ} \mathrm{C} \mathrm{min}^{-1}$ under nitrogen. Differential scanning calorimetry (DSC) 
thermograms were measured using an SII DSC6220 (Hitachi High-Tech Science) under nitrogen. The samples were first heated up to $100^{\circ} \mathrm{C}$ from room temperature and held for $5 \mathrm{~min}$ to eliminate the thermal history, then cooled down to $-100^{\circ} \mathrm{C}$ and held for $5 \mathrm{~min}$, and subsequently heated from -100 to $100{ }^{\circ} \mathrm{C}$. Both of the heating and cooling processes were operated at the same scanning rate of $10^{\circ} \mathrm{C} \mathrm{min}^{-1}$. A scanning electron microscope (SEM) analysis was carried out on a Hitachi SU-3500 instrument (Hitachi High-Technologies, Tokyo, Japan). Rheological measurements were performed on a Haake RheoStress 6000 Rheometer (Thermo Fisher Scientific) using $20 \mathrm{~mm}$ diameter parallel plates. To ensure the adhesion of the samples to the plates, an initial force of $25 \mathrm{~N}$ was employed. A stress sweep test was initially performed to ensure that the stress used was within the linear viscoelastic range. Frequency sweeps were executed on each of the samples over a frequency range of $0.1-100 \mathrm{rad} \mathrm{s}^{-1}$ at $80^{\circ} \mathrm{C}$.

\section{RESULTS AND DISCUSSION}

\section{Structural characterization of LPRA and BPRA}

LPRA was synthesized according to a previously reported method. ${ }^{19}$ The degree of polymerization of LPRA was $\sim 10$, which was calculated

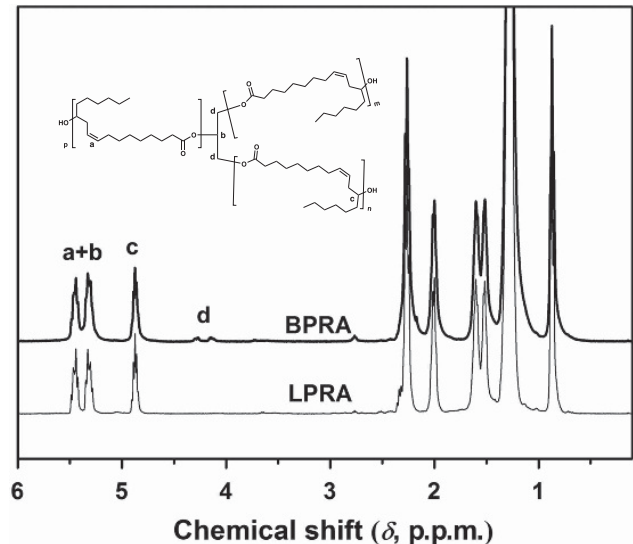

Figure $2{ }^{1} \mathrm{H}-\mathrm{NMR}$ spectra of LPRA and BPRA. BPRA, tri-branched poly (ricinoleic acid); LPRA, linear poly(ricinoleic acid); NMR, nuclear magnetic resonance. from ${ }^{1} \mathrm{H}$-NMR spectroscopic results (Figure 2). For the synthesis of BPRA, castor oil was used as a multi-functional initiator, which is biobased and well-compatible with ricinoleic acid; castor oil possesses $\sim 2.7$ hydroxyl groups per molecule. The introduction of castor oil was expected to not only increase the molecular weight but also lead to a branched structure. To promote the reaction toward the favored direction, the molar ratio between ricinoleic acid and castor oil was set as $40: 1$. By removing the unreacted monomer and oligomers, the product showed a unimodal peak in the size exclusion chromatography curve (Supplementary Figure S2). The molecular weight was $\sim 1.0 \times 10^{4} \quad(\mathrm{PDI}=1.8)$ and was higher than that of LPRA $\left(M_{\mathrm{w}}=6.6 \times 10^{3}\right.$, PDI $\left.=2.5\right)$. For the ${ }^{1} \mathrm{H}-\mathrm{NMR}$ spectrum of BPRA (Figure 2), characteristic peaks derived from castor oil were found in a range from 4.0 to 4.4 p.p.m. (peaks d). From the ratio of the integrated area between these peaks and peak c, due to an ester bond at 4.9 p.p.m., it was confirmed that every branch of BPRA was composed of $\sim 12$ monomers. Moreover, the synthesis of LPRA and BPRA was confirmed by the FT-IR spectroscopic analysis. The characteristic peaks for hydroxyl $\left(3200-3650 \mathrm{~cm}^{-1}\right)$ and carboxylate $\left(1710 \mathrm{~cm}^{-1}\right)$ groups that were found in the spectrum of ricinoleic acid disappeared in that of the product (Supplementary Figures S3 and S4), instead a strong peak owing to the ester carbonyl bond appeared at $1730 \mathrm{~cm}^{-1}$. The above spectroscopic data clearly support the formation of BPRA.

Morphology of EuTPI, EuTPI-LPRA and EuTPI-BPRA blend films LPRA and BPRA were separately introduced into EuTPI to examine their ability to improve the properties of EuTPI. For the EuTPI-LPRA blend films, a uniform appearance was observed when 5 and $10 \mathrm{wt} \%$ of LPRA was introduced. With increasing ratios of LPRA, the surface of the blend film became sticky, suggesting that a macro-phase separation occurred. Figure 3 shows the SEM images of the surface morphologies of the EuTPI-LPRA blend films. For EuTPI-LPRA5\%, small droplets with irregular shapes in the EuTPI matrix were

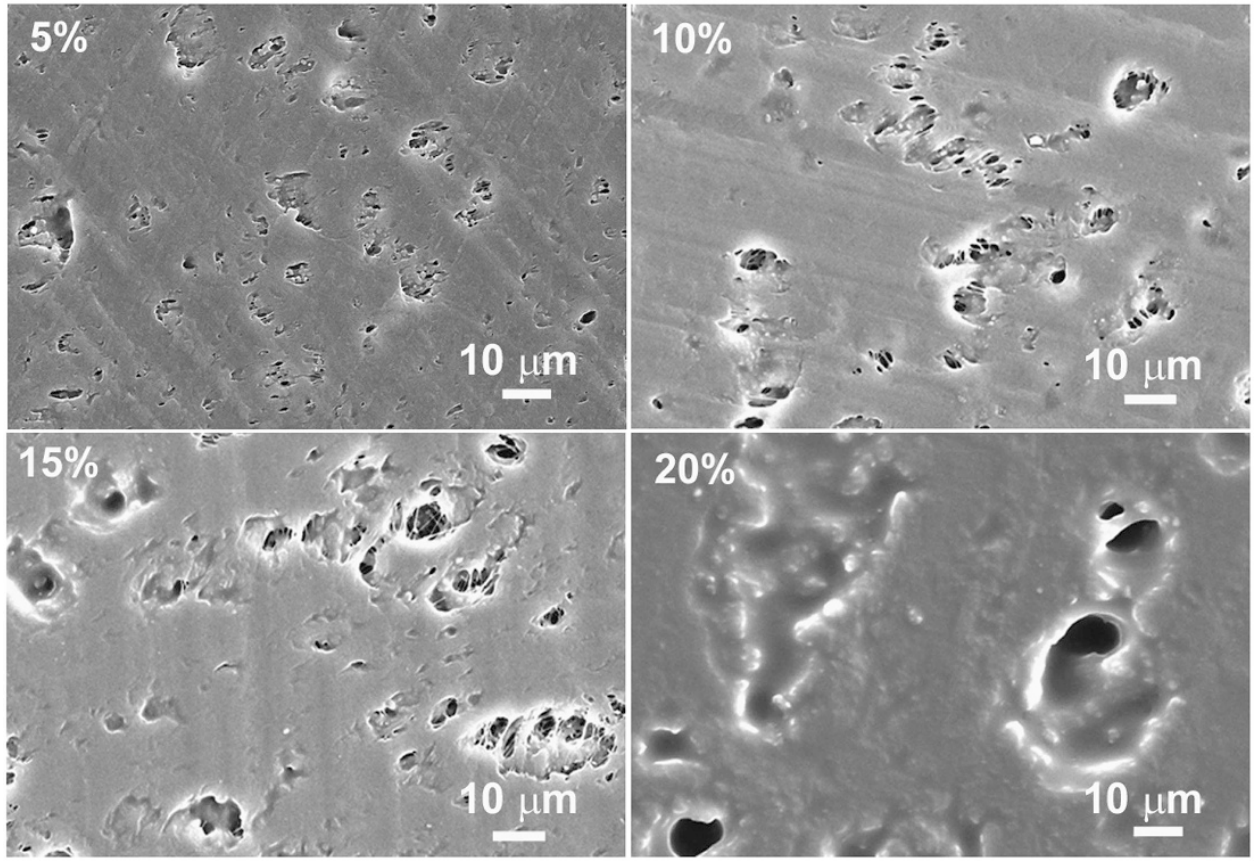

Figure 3 SEM micrographs of the surfaces of EuTPI-LPRA5\%, EuTPI-LPRA10\%, EuTPI-LPRA15\% and EuTPI-LPRA20\%. BPRA, tri-branched poly (ricinoleic acid); EuTPI, Eucommia ulmoides Oliver; LPRA, linear poly(ricinoleic acid); SEM, scanning electron microscope. 

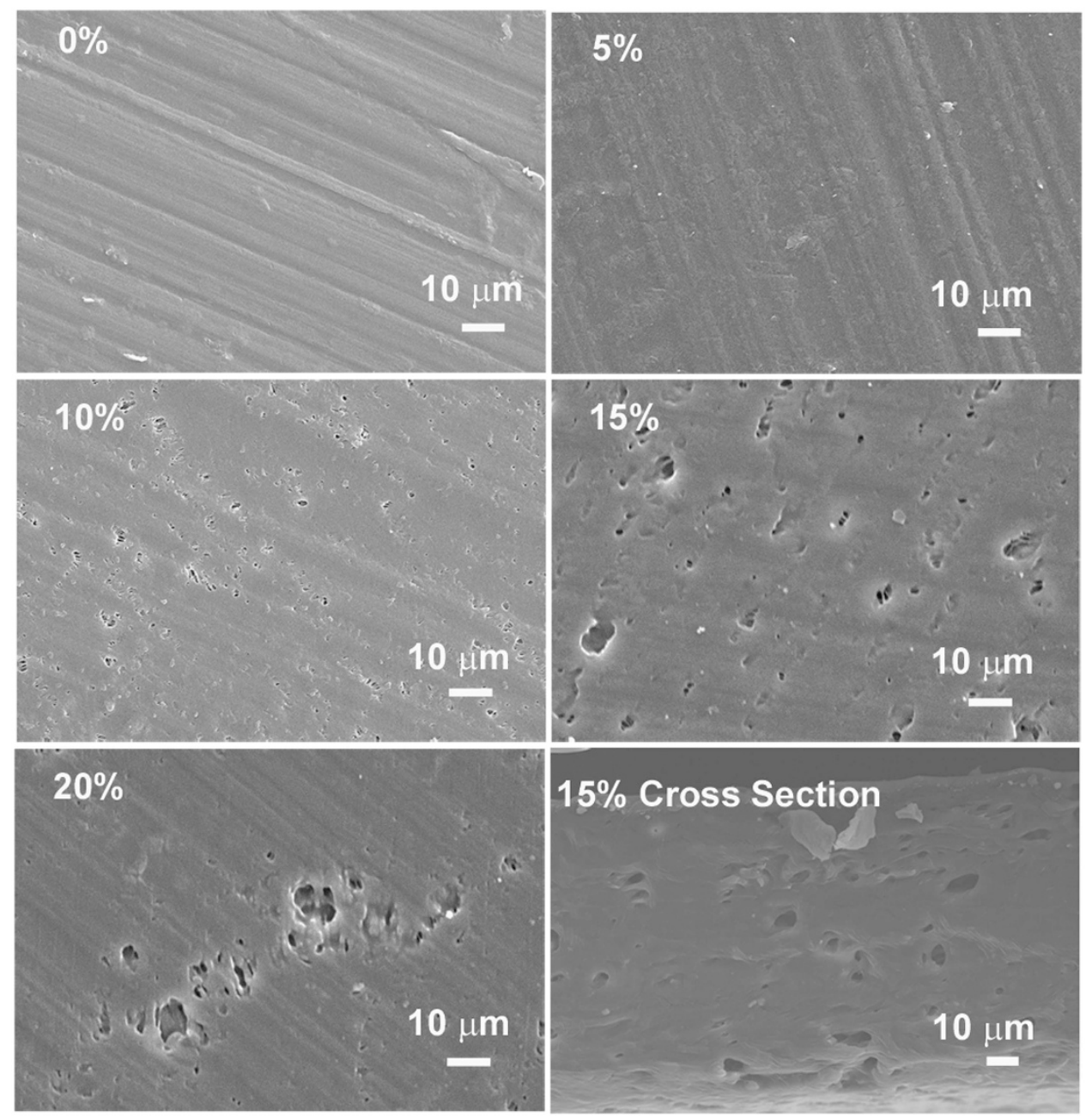

Figure 4 SEM micrographs of the surfaces of EuTPI, EuTPI-BPRA5\%, EuTPI-BPRA10\%, EuTPI-BPRA15\%, EuTPI-BPRA20\% and the cross-section of EUTPI-BPRA15\%. BPRA, tri-branched poly(ricinoleic acid); EuTPI, Eucommia ulmoides Oliver; SEM, scanning electron microscope.
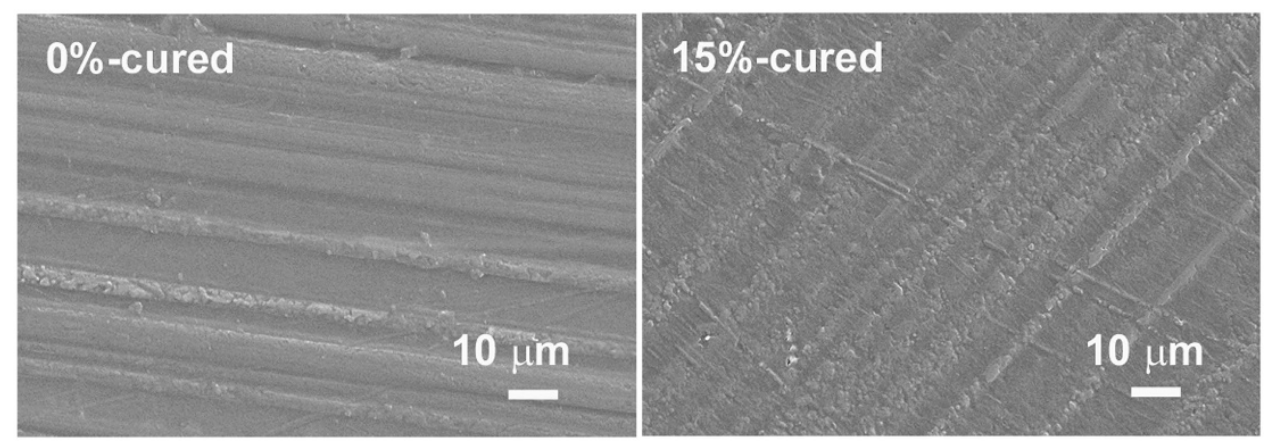

Figure 5 SEM micrographs of the surfaces of EuTPI-cured and EuTPI-BPRA15\%-cured samples. BPRA, tri-branched poly(ricinoleic acid); EuTPI, Eucommia ulmoides Oliver; SEM, scanning electron microscope.

observed. As the mixed ratio of LPRA increased, the droplets coalesced and were non-uniformly distributed in the matrix of EuTPI.

In contrast, all films consisting of EuTPI and BPRA showed a uniform appearance and a hard surface, suggesting that a macro-phase separation did not occur. Figure 4 shows the SEM micrographs of pure EuTPI and EuTPI-BPRA blend films. In the case of Eu-BPRA5\%, a similar morphology to pure EuTPI was observed, implying that BPRA was compatible with EuTPI. When $10 \mathrm{wt} \%$ of BPRA was added, small droplets appeared and were uniformly distributed in the EuTPI matrix. For blends up to $15 \mathrm{wt} \%$ of BPRA, the droplets presented a uniform distribution. An increasing size of the droplets was also observed not only on the surface but also in the cross-section. For EuTPI-BPRA20\%, however, the coalescence became severe, and the droplets were non-uniformly distributed. Therefore, a mixing of $15 \mathrm{wt}$ $\%$ BPRA should be the upper limitation for the blending. The above results indicate that BPRA possessed a better compatibility with EuTPI 

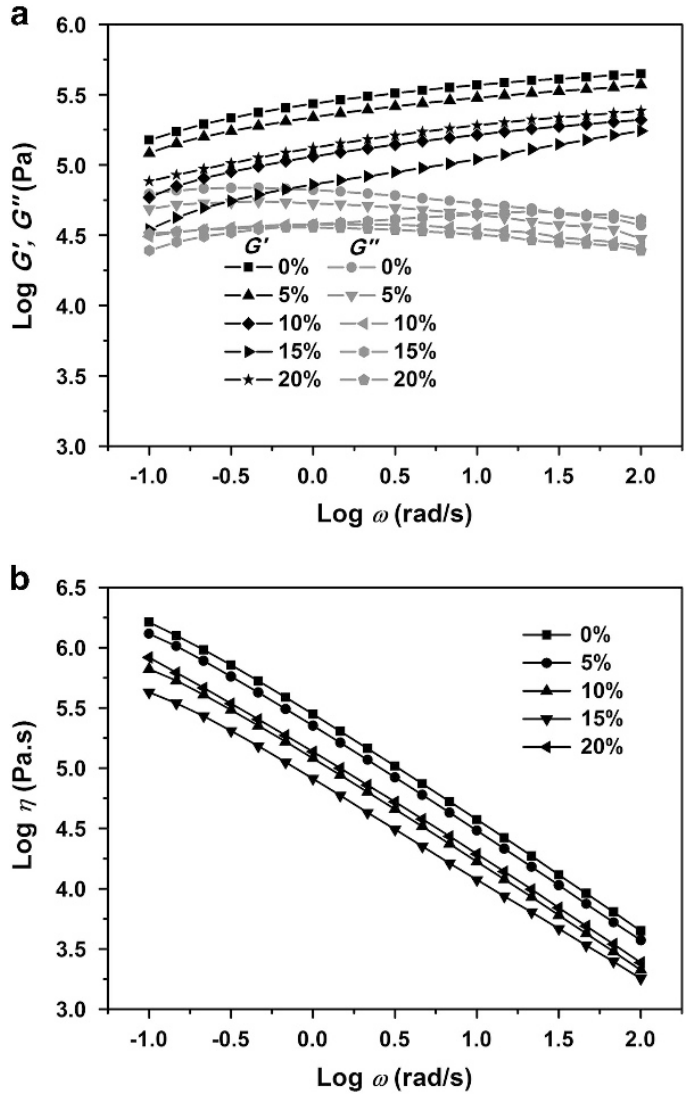

Figure 6 Rheological properties of EuTPI, EuTPI-LPRA5\%, EuTPI-LPRA10\%, EuTPI-LPRA15\% and EuTPI-LPRA20\%: (a) Frequencydependent dynamic storage modulus $G^{\prime}$ and loss modulus $G^{\prime \prime}$ curves; (b) Frequency-dependent complex viscosity $\eta$ curves. EuTPI, Eucommia ulmoides Oliver; LPRA, linear poly(ricinoleic acid).

than LPRA and could be uniformly distributed in the EuTPI matrix in the form of small droplets up to a content of $15 \mathrm{wt} \%$. It is believed that the obvious differences between EuTPI-LPRA and EuTPI-BPRA blend films were caused by the different interfacial tensions of EuTPI with LPRA and BPRA. ${ }^{27}$ The branched structure and more terminal hydroxyl groups of BPRA with respect to LPRA would result in a larger interfacial tension with the EuTPI matrix. The large interfacial tension led to the formation of small droplets of BPRA, which could steadily and uniformly exist in the EuTPI matrix up to a content of 15 wt $\%$. In contrast, LPRA with a smaller interfacial tension easily coalesced and was non-uniformly distributed in the EuTPI matrix.

The morphological change of the cured samples was further investigated. Figure 5 shows the surface morphologies of the EuTPIcured and EuTPI-BPRA15\%-cured samples. EuTPI-cured sample possessed a surface morphology similar to that of the pure EuTPI sample without crosslinking (Figure 4). For EuTPI-BPRA15\%-cured sample, however, the uniform droplets disappeared, displaying a homogenous surface compared with that of EuTPI-BPRA15\% without crosslinking. These SEM images suggest that BPRA was chemically incorporated into the crosslinked network of EuTPI through a reaction between the $\mathrm{C}=\mathrm{C}$ bonds of EuTPI and BPRA. Compared with other common additives, such as glycerol, BPRA was much more integrated in the EuTPI matrix, leading to a more stable performance of the final product.
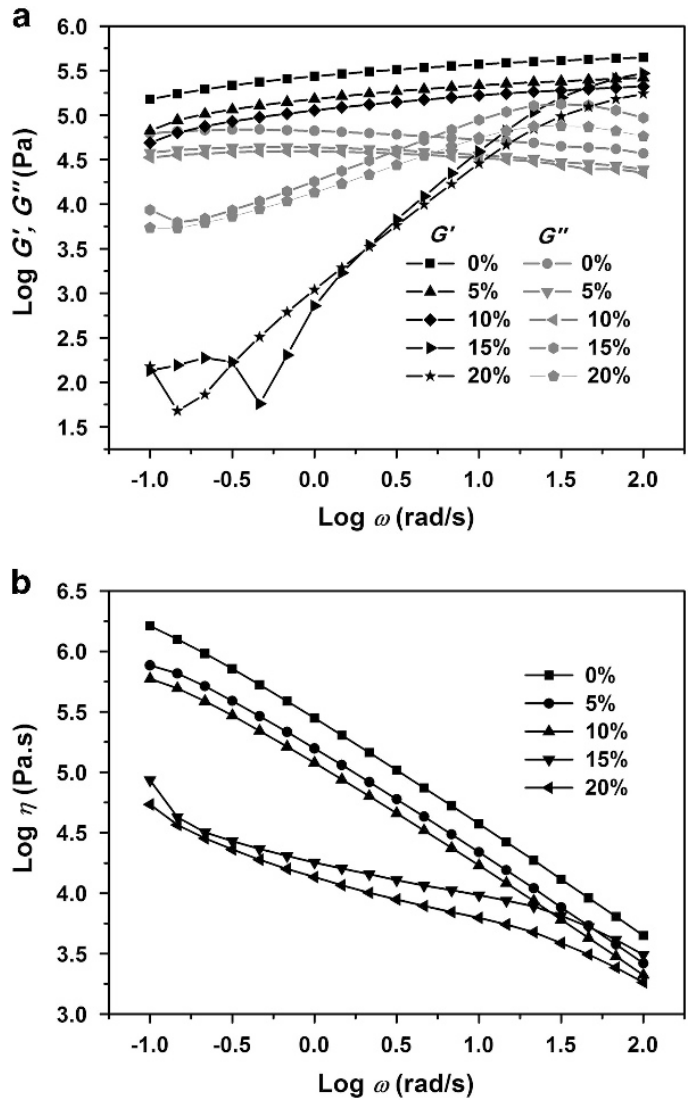

Figure 7 Rheological properties of EuTPI, EuTPI-BPRA5\%, EUTPI-BPRA10\%, EuTPI-BPRA15\% and EuTPI-BPRA20\%: (a) Frequencydependent dynamic storage modulus $G^{\prime}$ and loss modulus $G^{\prime \prime}$ curves; (b) Frequency-dependent complex viscosity $\eta$ curves. BPRA, tri-branched poly(ricinoleic acid); EuTPI, Eucommia ulmoides Oliver.

\section{Rheology studies}

The rheological properties of EuTPI, EuTPI-LPRA and EuTPI-BPRA blend films were studied by a rheometer. Figure 6 shows the results of EuTPI-LPRA blend films. The storage moduli $\left(G^{\prime}\right)$ and loss moduli $\left(G^{\prime \prime}\right)$ of all films displayed plateaus across the entire frequency range $(\omega)$ from 0.1 to $100 \mathrm{rad} \mathrm{s}^{-1}$ at $80^{\circ} \mathrm{C}$, suggesting a strong entanglement of molecular chains. By increasing the content of LPRA, $G^{\prime}, G^{\prime \prime}$ and the complex viscosity $(\eta)$ exhibited a tendency to decrease. For EuTPI-LPRA20\%, however, $G^{\prime}$ and $\eta$ were abnormally higher than those of EuTPI-LPRA10\% and EuTPI-LPRA15\%, which is probably due to the more severe coalescence and non-uniform distribution of LPRA in the EuTPI matrix. For all EuTPI-LPRA blend samples, $G^{\prime}$ was higher than the corresponding $G^{\prime \prime}$, implying the elastic gel behavior of EuTPI-LPRA blend samples at $80^{\circ} \mathrm{C}$. These data indicate that LPRA was not effective at improving the processability of EuTPI.

For EuTPI, EuTPI-BPRA5\% and EuTPI-BPRA10\%, $G^{\prime}$ was higher than $G^{\prime \prime}$, and all of them showed plateaus across the entire frequency range $(\omega)$ from 0.1 to $100 \mathrm{rad} \mathrm{s}^{-1}$ at $80^{\circ} \mathrm{C}$ (Figure $7 \mathrm{a}$ ). These results suggest the existence of a strong entanglement between molecular chains, which made the samples behave like elastic gels and lose mobility even above the melting point of EuTPI. Below $10 \mathrm{wt} \%$ of BPRA, $G^{\prime}, G^{\prime \prime}$ and $\eta$ only displayed a slight decrease with the increasing content of BPRA. As for EuTPI-BPRA15\% and EuTPIBPRA20\%, $G^{\prime}$ was lower than $G^{\prime \prime}$ at low frequency (Figures 7a and 8), displaying liquid behavior. Moreover, $G^{\prime}$ and $\eta$ were drastically reduced at low frequency. Taking EuTPI-BPRA15\% for instance, 

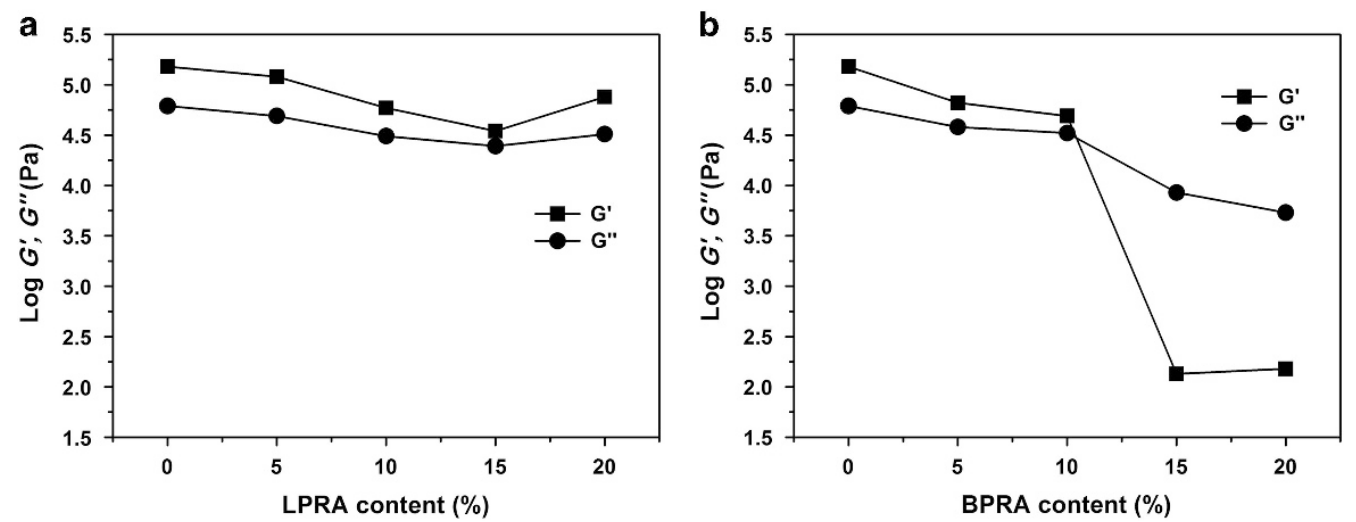

Figure 8 Dynamic storage modulus $G^{\prime}$ and loss modulus $G^{\prime \prime}$ values at $\omega=0.1 \mathrm{rad} \mathrm{s}^{-1}$ vary with the content of (a) LPRA and (b) BPRA. BPRA, tri-branched poly(ricinoleic acid); LPRA, linear poly(ricinoleic acid).
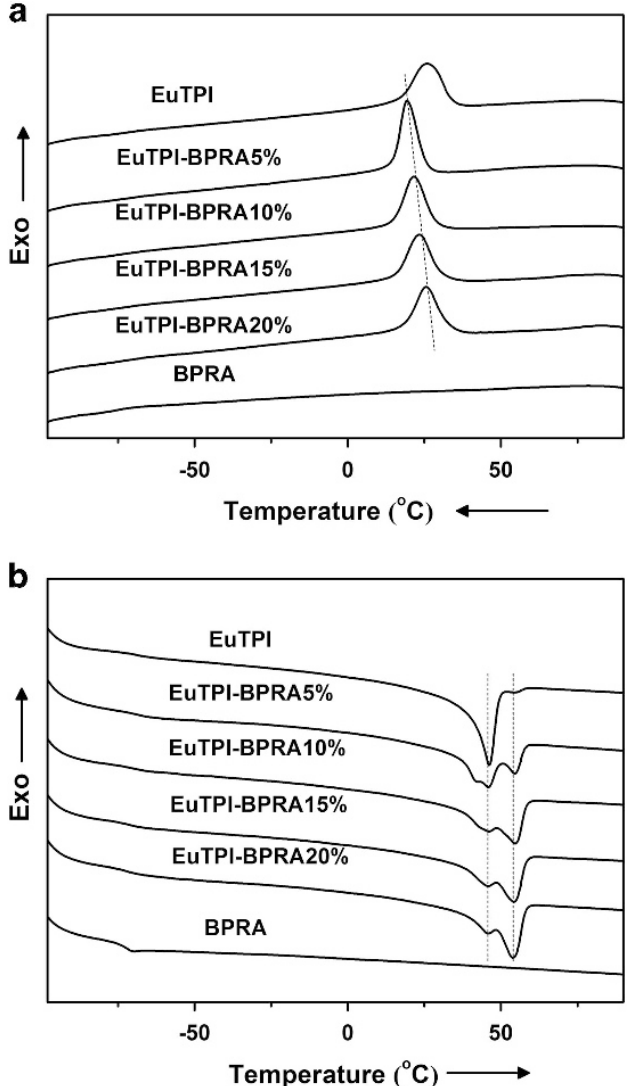

Figure 9 DSC traces of BPRA, EUTPI, EuTPI-BPRA5\%, EuTPI-BPRA10\%, EUTPI-BPRA15\% and EuTPI-BPRA20\%: (a) Cooling scan and (b) Subsequent heating scan. BPRA, tri-branched poly(ricinoleic acid); DSC, differential scanning calorimetry; EuTPI, Eucommia ulmoides Oliver.

$G^{\prime}$ was lowered nearly three magnitudes (Figure 8) and $\eta$ was reduced to $\sim 5 \%$ with respect to pure EuTPI at $\omega=0.1 \mathrm{rad} \mathrm{s}^{-1}$. The mechanical properties of the blends of immiscible materials were highly dependent on the morphology, such as the droplet size and the distance between the droplets. ${ }^{28}$ When a low content of BPRA was added, the BPRA dispersed in the EuTPI matrix in the form of small droplets, and the distance between the droplets was large. The mechanical properties of the EuTPI matrix were only slightly affected. When the BPRA content was increased, the size of the droplets
Table 1 Non-isothermal crystallization of EuTPI and EuTPI-BPRA blend samples

\begin{tabular}{lcccc}
\hline & $\mathrm{T}_{c}\left({ }^{\circ} \mathrm{C}\right)$ & $\chi_{c}(\%)$ & $\alpha^{{ }^{\mathrm{a}}}$ & $\beta \%^{\mathrm{a}}$ \\
\hline EuTPI & 26 & 32 & 2 & 98 \\
EuTPI-BPRA5\% & 20 & 28 & 15 & 85 \\
EuTPI-BPRA10\% & 22 & 26 & 31 & 69 \\
EuTPI-BPRA15\% & 23 & 26 & 33 & 67 \\
EuTPI-BPRA20\% & 26 & 24 & 37 & 63
\end{tabular}

Abbreviations: BPRA, tri-branched poly(ricinoleic acid); EuTPI, Eucommia ulmoides Oliver. aPercentage of the crystallinity of $\alpha$-phase and $\beta$-phase crystallites, respectively.

became larger and the distance between the droplets became smaller, resulting in the weakened mechanical strength of the EuTPI matrix. The entanglements between the EuTPI chains could be easily released at the low frequency, and the mobility was improved. Moreover, the small droplets of BPRA functioned as a lubricant to further improve the mobility of the EuTPI phase. This also explains the behavior transformation from elastic gel to liquid with the increase of BPRA above the melting point of EuTPI. When $20 \mathrm{wt} \%$ of BPRA was added, however, the coalescence became extensive (Figure 4 ). Therefore, $\sim 15$ wt $\%$ of BPRA would be appropriate for the practical application as a processing aid for EuTPI.

The above results indicate that BPRA was more effective at improving the processability of EuTPI compared with LPRA because BPRA possessed a better compatibility with EuTPI and could exist in the EuTPI matrix as uniform droplets up to a content of $15 \mathrm{wt} \%$.

\section{Thermal properties}

The thermal stability of EuTPI, BPRA and EuTPI-BPRA15\% was investigated by Thermogravimetric analysis. The results (Supplementary Figure S5) show that both EuTPI and BPRA possessed a good thermal stability. The decomposition temperatures $\left(T_{\mathrm{d} 5}\right)$ of EuTPI and BPRA were $348^{\circ} \mathrm{C}$ and $329^{\circ} \mathrm{C}$, respectively. For EuTPI-BPRA15\%, $T_{\mathrm{d} 5}$ was $\sim 338^{\circ} \mathrm{C}$, which indicated that the introduction of BPRA would not compromise the thermal stability of EuTPI during processing and usage.

The crystallization behaviors of EuTPI, EuTPI-LPRA and EuTPI-BPRA blend samples were investigated by DSC. The crystallinity was calculated based on the reported melting enthalpies for $100 \%$ pure $\alpha$-form and $\beta$-form crystals of trans-1,4-polyisoprene, which are $12.8 \mathrm{KJ} \mathrm{mol}^{-1}$ and $9.6 \mathrm{KJ} \mathrm{mol}^{-1}$ per repeat unit, respectively. ${ }^{9}$ 
LPRA and BPRA were completely amorphous because the regular arrangement was disturbed by the dangling chains of the polymer. EuTPI showed a crystallization peak at $26^{\circ} \mathrm{C}$ during the cooling scan (Figure 9a). Two endothermic peaks located at $46^{\circ} \mathrm{C}$ and $54^{\circ} \mathrm{C}$ were observed during the heating process (Figure $9 \mathrm{~b}$ ), which correspond to the transformation from $\beta$ to $\alpha$ phases and the melting of the $\alpha$ phase, respectively. ${ }^{2,29-31}$ BPRA and LPRA possessed similar effects on the crystallization of EuTPI (Figure 9 and Supplementary Figure S6, Table 1 and Supplementary Table S1). Considering the non-uniform distribution of LPRA in the EuTPI matrix, the influence of BPRA on the crystallization behaviors of EuTPI was mainly discussed. As shown in Table 1, the crystallinity $\left(\chi_{\mathrm{c}}\right)$ of EuTPI rapidly decreased from 32 to $28 \%$ with the addition of $5 \mathrm{wt} \%$ of BPRA and then displayed a small decrease on further increasing the content of BPRA. The blend film still retained a high crystallinity (24\%) when $20 \mathrm{wt} \%$ of BPRA was introduced. It is believed that BPRA possessed two different effects on the crystallization of EuTPI with respect to spatial distribution. Only a small part of BPRA was located in the EuTPI phase because of the poor miscibility between BPRA and EuTPI. This part of BPRA functioned as an impurity, disturbing the regular arrangement of EuTPI chains. Most of the BPRA existed as separated droplets. These droplets exerted less influence on the crystallization of EuTPI.

It is worthy to note that with an increase in the content of BPRA, the relative amount of $\alpha$-phase crystallites first rapidly increased from 2 to $31 \%$ and finally reached $37 \%$ (Table 1). As mentioned above, BPRA existed as two parts: one existed in the EuTPI phase and the other existed as isolated droplets. It was the former part rather than the latter part that contributed to the increase of $\alpha$-phase crystallites. Although the former part would suppress the total crystallinity, it could improve the mobility of the EuTPI chains and favor the formation of more stable $\alpha$-phase crystallites because of the high mobility and flexibility of BPRA.

\section{CONCLUSION}

In this study, LPRA and BPRA were synthesized using an immobilized lipase as the catalyst, and each polymer was separately introduced into EuTPI to improve the processability. BPRA possessed better compatibility with EuTPI compared with LPRA and could exist in the EuTPI matrix as uniform droplets up to a content of $15 \mathrm{wt} \%$, leading to an obvious improvement in the processability of EuTPI. BPRA could also be chemically incorporated into the crosslinked network of EuTPI through a reaction between the $\mathrm{C}=\mathrm{C}$ bonds of EuTPI and BPRA, which hindered BPRA from escaping the EuTPI matrix and contributed to the stable performance of the final products. BPRA could serve as a crosslinkable processing aid for EuTPI.

\section{CONFLICT OF INTEREST}

The authors declare no conflict of interest.

\section{ACKNOWLEDGEMENTS}

B-xZ thanks the China Scholarship Council (CSC) for a scholarship. This study was partly supported by the Science and Technology Research Promotion Program for Agriculture, Forestry, Fisheries and Food Industry from the Ministry of Agriculture, Forestry and Fisheries of Japan.

1 Kent, E. G. \& Swinney, F. B. Properties and applications of trans-1,4-polyisoprene. Ind. Eng. Chem. Prod. Res. Dev. 5, 134-138 (1966).
2 Goodman, A., Schilder, H. \& Aldrich, W. The thermomechanical properties of guttapercha: II. The history and molecular chemistry of gutta-percha. Oral. Surg. Oral. Med. Oral. Pathol. 37, 954-961 (1974).

3 Nakazawa, Y., Takeda, T., Suzuki, N., Hayashi, T., Harada, Y., Bamba, T. \& Kobayashi, A. Histochemical study of trans-polyisoprene accumulation by spectral confocal laser scanning microscopy and a specific dye showing fluorescence solvatochromism in the rubber-producing plant, E. ulmoides Oliver. Planta 238 549-560 (2013).

4 Tangpakdee, J., Tanaka, Y., Shiba, K., Kawahara, S., Sakurai, K. \& Suzuki, Y. Structure and biosynthesis of trans-polyisoprene from Eucommia ulmoides. Phytochemistry 45, 75-80 (1997).

5 Nakazawa, Y., Bamba, T., Takeda, T., Uefuji, H., Harada, Y., Li, X., Chen, R., Inoue, S., Tutumi, M., Shimizu, T., Su, Y., Gyokusen, K., Fukusaki, E. \& Kobayashi, A. Production of Eucommia-rubber from Eucommia ulmoides Oliv. (Hardy Rubber Tree). Plant Biotechnol. 26, 71-79 (2009).

6 Bamba, T., Fukusaki, E., Nakazawa, Y. \& Kobayashi, A. In-situ chemical analyses of trans-polyisoprene by histochemical staining and Fourier transform infrared microspectroscopy in a rubber-producing plant, Eucommia ulmoides Oliver. Planta 215, 934-939 (2002).

7 Arvanitoyannis, I., Kolokuris, I., Nakayama, A. \& Aiba, S. Preparation and study of novel biodegradable blends based on gelatinized starch and 1,4-trans-polyisoprene (gutta percha) for food packaging or biomedical applications. Carbohydr. Polym. 34, 291-302 (1997).

8 Tsujimoto, T., Toshimitsu, K., Uyama, H., Takeno, S. \& Nakazawa, Y. Maleated trans-1,4-polyisoprene from Eucommia ulmoides Oliver with dynamic network structure and its shape memory property. Polymer 55, 6488-6493 (2014).

9 Zhang, J. \& Xue, Z. A comparative study on the properties of Eucommia ulmoides gum and synthetic trans-1,4-polyisoprene. Polym. Test. 30, 753-759 (2011).

10 Xue, Z., Zhang, J., Li, X. \& Yan, R. Damping properties of Eucommia ulmoides gum and its blends. Int. Symp. Eucommia ulmoides 1, 116-118 (2007).

11 Yan, R. Insight into materials engineering of Eucommia ulmoides gum. Int. Symp. Eucommia ulmoides 1, 34-37 (2007).

12 Sarina, Zhang, J. \& Zhang, L. Dynamic mechanical properties of Eucommia ulmoides gum with different degree of cross-linking. Polym. Bull. 68, 2021-2032 (2012)

13 Ogunniyi, D. S. Castor oil: A vital industrial raw material. Bioresour. Technol. 97, 1086-1091 (2006).

14 Mutlu, H. \& Meier, M. A. R. Castor oil as a renewable resource for the chemical industry. Eur. J. Lipid Sci. Technol. 112, 10-30 (2010).

15 Lebarbe, T., Ibarboure, E., Gadenne, B., Alfos, C. \& Cramail, H. Fully bio-based poly(l-lactide)-b-poly(ricinoleic acid)-b-poly(I-lactide) triblock copolyesters: investigation of solid-state morphology and thermo-mechanical properties. Polym. Chem. 4, 3357-3369 (2013).

16 Robertson, M. L., Paxton, J. M. \& Hillmyer, M. A. Tough blends of polylactide and castor oil. ACS Appl. Mater. Interfaces 3, 3402-3410 (2011).

17 Gautrot, J. E. \& Zhu, X. X. High molecular weight bile acid and ricinoleic acid-based copolyesters via entropy-driven ring-opening metathesis polymerisation. Chem. Commun. 0, 1674-1676 (2008).

18 Ebata, H., Yasuda, M., Toshima, K. \& Matsumura, S. Poly (ricinoleic acid) based novel thermosetting elastomer. J. Oleo Sci. 57, 315-320 (2008).

19 Ebata, H., Toshima, K. \& Matsumura, S. Lipase-catalyzed synthesis and curing of high-molecular-weight polyricinoleate. Macromol. Biosci. 7, 798-803 (2007).

20 Shikanov, A. \& Domb, A. J. Poly(sebacic acid-co-ricinoleic acid) biodegradable injectable in situ gelling polymer. Biomacromolecules 7, 288-296 (2006).

21 Kelly, A. R. \& Hayes, D. G. Lipase-catalyzed synthesis of polyhydric alcohol-poly (ricinoleic acid) ester star polymers. J. Appl. Polym. Sci. 101, 1646-1656 (2006).

22 Slivniak, R., Langer, R. \& Domb, A. J. Lactic and ricinoleic acid based copolyesters stereocomplexation. Macromolecules 38, 5634-5639 (2005).

23 Slivniak, R. \& Domb, A. J. Macrolactones and polyesters from ricinoleic acid. Biomacromolecules 6, 1679-1688 (2005).

24 Bódalo-Santoyo, A., Bastida-Rodríguez, J., Máximo-Martín, M. F., Montiel-Morte, M. C. \& Murcia-Almagro, M. D. Enzymatic biosynthesis of ricinoleic acid estolides. Biochem. Eng. J. 26, 155-158 (2005).

25 Teomim, D., Nyska, A. \& Domb, A. J. Ricinoleic acid-based biopolymers. J. Biomed. Mater. Res. 45, 258-267 (1999).

26 Krasko, M. Y., Shikanov, A., Ezra, A. \& Domb, A. J. Poly(ester anhydride)s prepared by the insertion of ricinoleic acid into poly(sebacic acid). J. Polym. Sci. A Polym. Chem. 41, 1059-1069 (2003).

27 Jannerfeldt, G., Boogh, L. \& Manson, J. A. E. The morphology of hyperbranched polymer compatibilized polypropylene/polyamide 6 blends. Polym. Eng. Sci. 41, 293-300 (2001).

28 Chang, K., Robertson, M. L. \& Hillmyer, M. A. Phase inversion in polylactide/soybean oil blends compatibilized by poly(isoprene- $b$-lactide) block copolymers. ASC Appl. Mater. Interfaces 1, 2390-2399 (2009).

29 Anandakumaran, K., Kuo, C. C., Mukherji, S. \& Woodward, A. E. Crystallization of trans-1,4-polyisoprene. J. Polym. Sci. Polym. Phys. 20, 1669-1676 (1982).

30 Cooper, W. \& Vaughan, G. Crystallization of gutta percha and synthetic trans-1,4-Polyisoprenes. Polymer 4, 329-340 (1963).

31 Pathak, A., Saxena, V., Tandon, P. \& Gupta, V. D. Vibrational dynamics of trans-1,4-polyisoprene ( $\beta$-form). Polymer 47, 5154-5160 (2006). 